\title{
CONTROLE A DISTANCE ET AUTOMATICITÉ DES INSTALLATIONS ÉLECTRIQUES
}

\author{
Édouard TAVERNIER \\ Ingénieur 1. E. G. \\ Ingénieur en chef du service installations \\ des Établissements Merlin \& Gerin
}

\section{INTRODUCTION}

Les constructeurs qui se sont penchés sur les problèmes soulevés par l'aulomaticité ou la télécommande des installations électriques, se sont ingéniés à mettre au point des solutions relativement simples, offrant les meilleures garanties de bon fonctionnement.

Dans la mesure où ils y sont parvenus, de telles réalisations sont loin d'entraîner des dépenses aussi élevées qu'on pourrait l'imaginer de prime abord, et nombreux sont les cas où ces dépenses peuvent être rapidement amorties par les: úconomies de toutes sortes qu'elles permettent de réaliser. Ce fait se vérifie notamment pour les installations de faible ou moyenne importance.

Les solutions décrites au cours de cet article permettront au lecteur de se' familiariser avec les principes de quelques dispositifs assez couramment employés.

Il ne faut cependant jamais perdre de vue que, seule, l'intelligence humaine, cuverte à tous les faits concrets, est apte à juger immédiatement des conséquences qui découlent d'un incident quelconque dans le fonctionnement normal d'une installation, et d'en déduire une ligne de conduite en vue d'une intervention plus ou moins rapide.

Il existe donc une différence fondamentale entre une exploitation placée directement sous son contrôle et une exploitation similaire commandée, en aehors de toute surveillance humaine, par une sorte de « robot» qui accomplit aveuglément toutes les tâches qui lui ont été préalablement assignées, quelles que soient les conjonctures du moment et quelles qu'en puissent être les conséquences.

Bien que d'apparence à priori assez simple, l'étude rationnelle de telles réalisations est souvent extrêmement délicate. L'homme raisonne toujours plus difficilement dans l'abstrait que devant un fait matériel, et pour la conception du «robot» proprement dit, la difficulté n'est pas d'imaginer ce que ce robot doit faire pour que les fonctions qui lui sont confiées s'accomplissent automatiquement, mais plutôt d'établir comment son intervention doit se modifier au cas où les conditions initialement prévues ne sont pas exactement remplies.

Il est essentiel, en un mot, de prévoir le comportement de notre «robol» devant un incident quelconque susceptible d'affecter plus ou moins gravement le fonctionnement correct de l'ensemble, et notamment devant les conséquences possibles d'une défaillance d'organes ou d'appareils survenant en dehors de toute surveillance humaine. C'est à cette condition seulement que de sérieux déboires peuvent être évités et que des réalisations de ce genre sont susceptibles d'apporter en toute sécurité à leurs exploitants les satisfactions qu'ils en allendent.

Leur élude rationnelle exige donc une connaissance approfondie de ce genrc d'installations, ainsi que des caractéristiques bien particulières du matériel afin d'en déceler les faiblesses et d'y parer par des dispositifs de sécurité appropriés. 


\section{GENERALITÉS SUR LES INSTALLATIONS} AUTOMATIQUES OU TÉLÉCOMMANDÉES

La surveillance et l'exploitation des installations d'une certaine importance, telles que centrales, postes répartiteurs, postes de transformation, exigent le plus souvent un personnel nombreux et qualifié. Ces installations fonctionnent généralement en permanence, et plusieurs équipes complètes doivent être constituées pour assurer la continuité du service, tout en réservant au personnel les périodes indispensables et légales de repos, résultant notamment de la législation en vigueur.

D'autres installations de moindre importance, telles que certains postes de transformation, par exemple, fonctionnent sans surveillance permanente. Cependant la recherche de meilleures conditions économiques d'exploitation, c'est-à-dire du meilleur rendement, conduit à proportionner le nombre d'unités en service à l'appel d'énergie du réseau ou de l'usine qu'ils alimentent.

$D$ 'autres installations encore, notamment certains postes répartiteurs, pourraient fonctionner habituellement sans surveillance si certaines mancuvres d'isolement et de com- mutation pouvaient s'effectuer automatiquement ou être commandées à distance lorsqu'il faut éliminer un tronçon de ligne défectueux en rétablissant dans le plus bref délai, partout où cela reste possible, le courant sur les sections du réseau restées saines.

On est donc souvent amené à envisager, pour certaines de ces installations :

- soit la signalisation et la commande de leurs machines, organes ou appareils, depuis une centrale, un poste répartiteur ou un poste central de commande plus ou moins éloignés et normalement surveillés;

- soit l'automaticité pure et simple du fonctionnement de leurs divers organes ou machines en fonction des exigences multiples et momentanées du réseau et des conditions d'exploitation rationnelles de l'ensemble;

- soit, le plus généralement, une solution mixte comportant une semi-automaticité de certains organes, combinée avec la signalisation et la commande à distance des seules fonctions essentielles.

Il devient alors possible de supprimer toute surveillance permanente de telles installations, lorsque les distances qui les séparent des installations voisines normale-

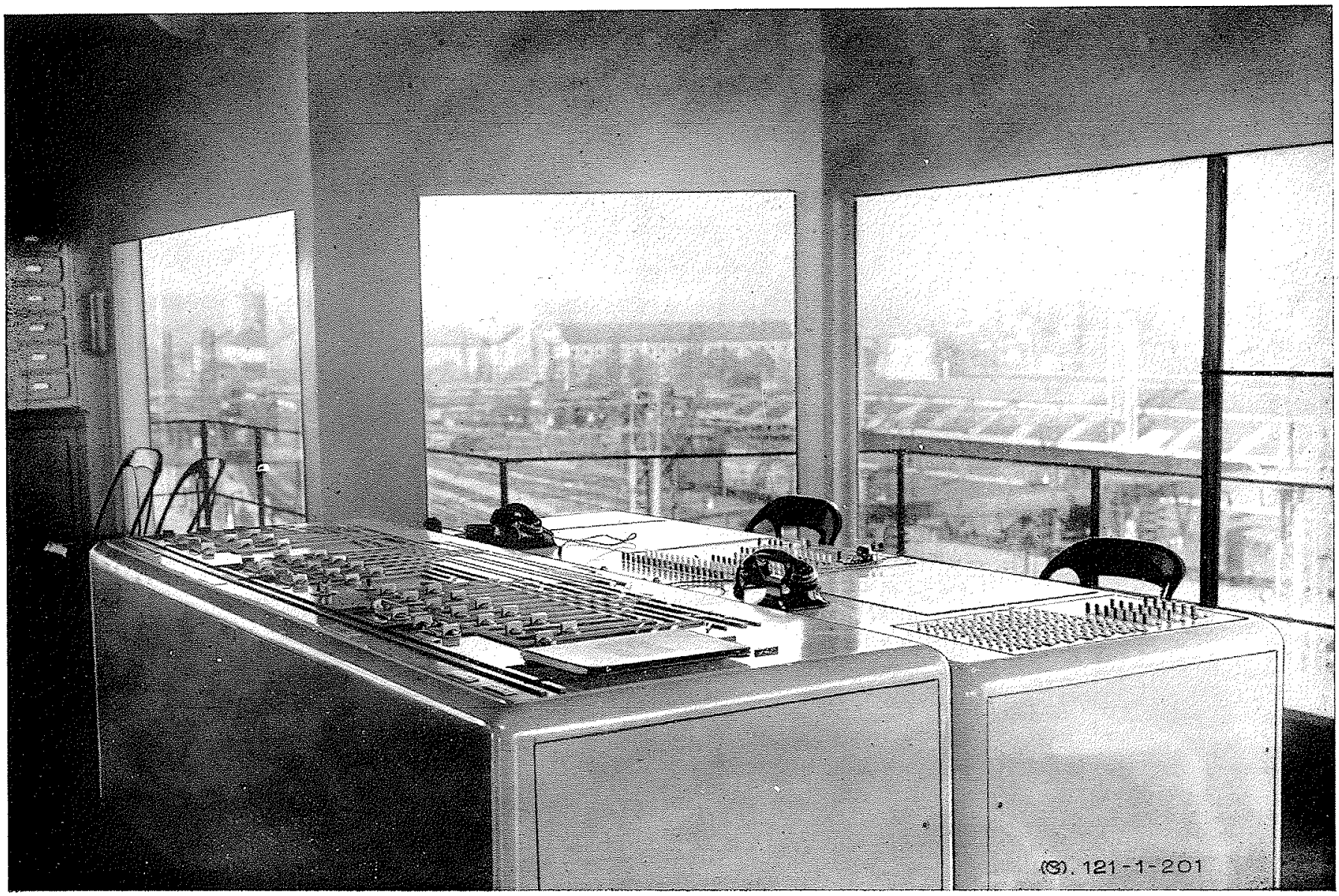

POSTE DE COMMANDE A DISTANCE, DE LA GARE DU MANS 
ment surveillées sont relativement faibles. Pour les distances plus importantes, on prévoit généralement un simple gardien qui, en dehors de quelques visites périodiques et travaux d'entretien courant, peut consacrer la majeure partie de son temps à ses occupations familiales.

Nous examinerons rapidement, au cours de cet article, quelques solutions les plus couramment employées pour parvenir à ce résultat. D'une manière générale, ces réalisations doivent être minutieusement étudiées et exécutées. Le matériel employé doit être robuste et de toute première qualité. Des dispositifs de sécurité judicieusement établis seront prévus pour intervenir dans le sens convenable en cas de dérangement ou d'avarie afin d'éviter toutes détériorations aux machines ou appareils en attendant l'arrivée du personnel qualifié chargé de remettre l'installation en état de marche.

Il n'est pas possible de retenir à priori l'un quelconque des dispositifs décrits plus loin comme étant la meilleure solution à la généralité des problèmes posés. Le choix d'un dispositif, dans chaque cas particulier, dépend essentiellement de nombreuses considérations, telles que la position géographique de l'installation, la distance qui la sépare d'autres installations les plus voisines, la nature de ces dernières, les particularités techniques et les conditions habituelles d'exploitation.

Ce choix dépend également des considérations économiques habituelles qui impliquent de mettre en balance, d'une part les frais supplémentaires engagés par rapport à une installation normalement surveillée, d'autre part les économies de toutes sortes, notamment sur le personnel, qu'il sera possible de réaliser de ce fait.

\section{Dispositirs de protection et de sécurité des installations automativues ou télécommandées}

L'adoption de tels dispositifs est rendue plus impérative que dans une installation normalement surveillée du fait de l'impossibilité d'intervenir immédiatement en cas de dérangement ou d'avarie survenant aux machines, transformateurs ou appareils. Ces dispositifs sont habituellement les nêmes que ceux utilisés couramment dans les installations ordinaires. Ils doivent nécessairement comporter un système de voyants ou lampes indicatrices de défaut permettant au personnel chargé de la remise en service d'identifier la cause du dérangement dès son arrivée sur les lieux. Il est nécessaire également de prévoir un système avertisseur à distance signalant soit au gardien, soit à l'ćlectricien de service de l'installation voisine chargée du contrôle, qu'un incident vient de se produire et qu'il est nécessaire d'intervenir. Le schéma de la figure $\mathrm{n}^{\circ} \mathrm{I}$ ci-contre indique le principe de cette signalisation, le contact C. S. étant, par exemple, celui du thermomètre constatant l'échauffement d'un palier d'une machine.

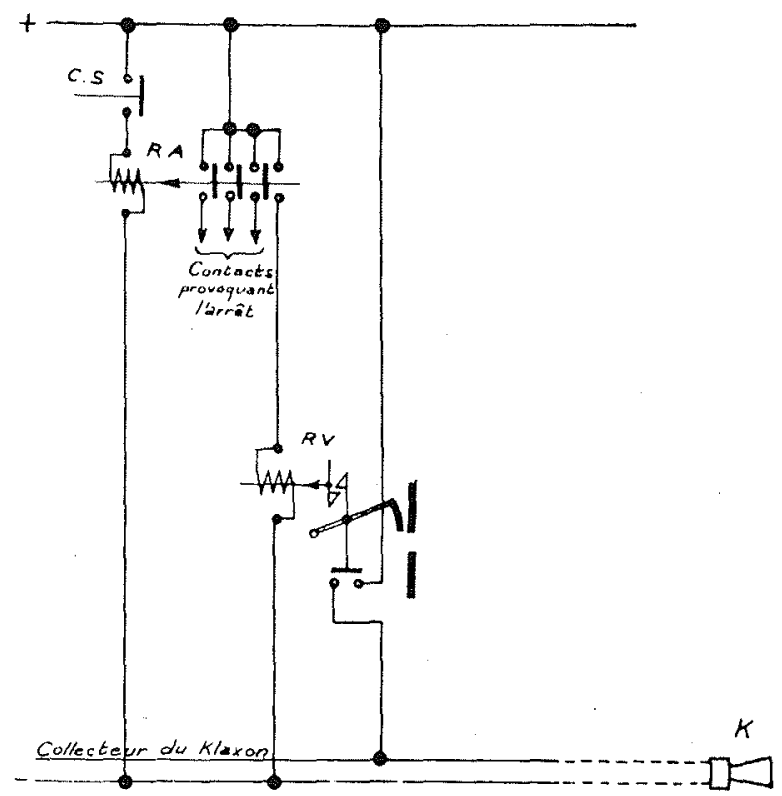

FIG. r. - Principe d'un dispositif de protection et dalarme CS: contact de sécurité. - RA : relais auxiliaire. $\mathrm{RV}$ : relais à voyant. - $\mathrm{K}$ : klaxon. - \pm : source atuiliaire

Parmi les dispositifs de sécurité que l'on rencontre le plus couramment, on peut citer notamment ceux conçus pour intervenir dans les hypothèses suivantes:

\section{Pour une turbine hydraulique :}

Baisse anormale du niveau d'eau au barrage,

Baisse anormale de pression dans les conduites,

Chute de courroie du tachymètre ou baisse anormale de tension de la dynamo tachymétrique,

Baisse anormale de la pression de l'huile du régulateur, Emballement,

E.chauffement des paliers;

Pour un alternateur :

Echauffement des paliers,

Echauffement des enroulements,

Protection contre l'incendie,

Défauts électriques, tels que mise à la masse des enroulements, courts-circuits entre phases, courts-circuits entre spires, retour d'énergie, maxima de tension, maxima d'intensité;

\section{Pour un transformateur :}

Défauts internes constatés par relais Buccholz, protections différentielles, protections de masse,

Echauffement anormal constaté par thermomètre à contacts; 
Pour l'ensemble des installations :

Claquage d'un câble,

Interruption de la source auxiliaire locale ou baisse anormale de la tension de cette source,

Coupure d'un circuit auxiliaire essentiel,

Dérangement dans le fonctionnement des appareils, relais ou circuits de commande, etc...

S'il s'agit d'un incident ne constituant pas un danger immédiat pour l'installation, on peut se borner à actionner le signal avertisseur sans provoquer l'arrêt automatique des organes correspondants. Il en est ainsi notamment du premier contact des relais Buccholz signalant un très faible dégagement gazeux, ou du premier contact d'un thermomètre de transformateur signalant une température anormale mais non encore dangereuse.

Pour ce qui concerne plus spécialement les dérangements susceptibles de se produire dans une installation automatique, on est souvent amené à établir des dispositifs de sécurité indépendants des circuits d'asservissement proprement dits, basés notamment sur la prédétermination du temps raisonnablement nécessaire à l'exécution d'un « cycle de mancurres $\gg(1)$.

Les circuits d'asservissement doivent être établis de telle sorte qu'une manœurre de rang $n$ ne puisse débuter que si la manœuvre de rang $n-1$ s'est correctement effectuée.

Les circuits de sécurité doivent agir pour ramener l'ensemble des organes au repos. Ils interviennent dans ce sens, au bout d'un temps légèrement supérieur à la durée normale du cycle de manœuvre, et uniquement dans le cas où, pour une raison quelconque, la manæuvre ne s'est pas correctement effectuée dans les délais prédéterminés. Ils actionnent parallèlement le dispositif avertisseur.

\section{INSTALLATIONS TÉLÉCOMMANDÉES}

Au delà de quelques centaines de mètres il devient difficile de contrôler et de commander une installation à distance en allongeant démesurément les circuits ordinaires de mesure, de signalisation et de commande.

Le prix des canalisations de liaison devient rapidement

(I) Par " cycle de mancurres » il faut entendre l'ensemble des opérations qui doivent stccessivement s'elfectuer dans un ordre déterminé denuis linpulsion initiale de commande jusqu'à la phase finale qui termine l'intervention du dispositif d'autonaticité. A titre d'exemple on peut citer la mise en route d'une turbine qui nécessite habituellement :

- la mise en pression du régulateur;

- la mise en pression du dispositif de graissage

- l'ouverture de la vanne de garde.

- l'action progressive dans le sens “ ouverture» du régulateur de vitesse juscu'à ce que la vitesse de régime soit atteinte. prohibitif et les chutes de tension dans les conducteurs basse-tension de faible section atteignent très vite des valeurs inacceptables.

On est ainsi conduit à rechercher des solutions qui réduisent au minimum la section et le nombre de fils pilotes indispensables, et pour de grandes distances, à supprimer totalement toutes les canalisations auxiliaires basse-tension entre l'installation contrôlée à distance et le lieu à partir duquel s'effectue ce contrôle.

Une installation de ce genre comporte :

- une télésignalisation donnant à chaque instant la position exacte de ses appareils ou organes principaux, tels que disjoncteurs, interrupteurs, sectionneurs d'aiguillage; le cas échéant, des indications diverses telles que position des vannes, fin de course des organes, hauteurs d'eau au barrage, etc..., ainsi que le système avertisseur en cas de dérangement ou de défaui;

- un système de télécommande permettant à volonté d'ouvrir ou de fermer les vannes, de mettre en route ou d'arrêter les machines, d'ouvrir ou de fermer les disjonc-

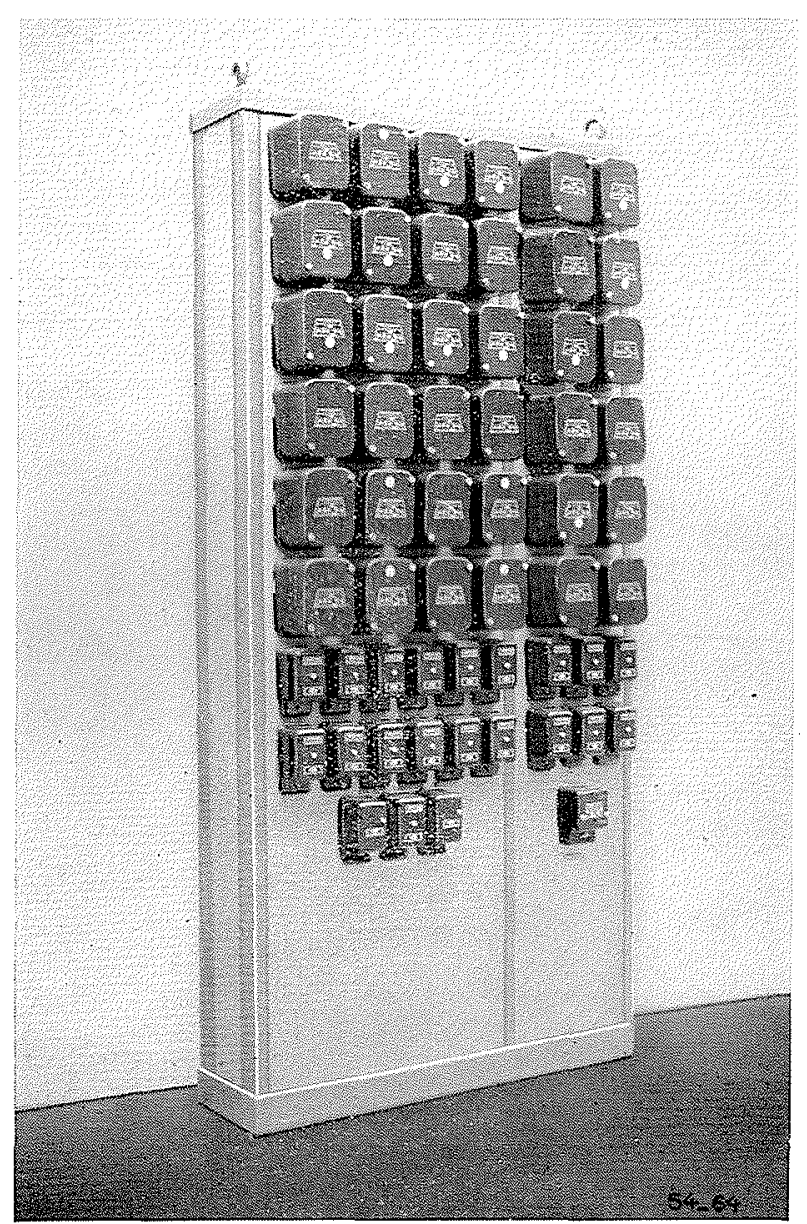

UN DES CHASSIS DE RELAIS DE TÉLÉCOMMANDE DE LA CENTRALE D'ORGEIX 
teurs, les sectionneurs principaux, en un mot d'effectuer toutes les mancurres courantes nécessitées par l'exploitation rationnelle de l'installation;

- l'ensemble est souvent complété par un système de télémesures reproduisant au poste de commande les indications données par les appareils de mesures essentiels de l'installation.

Il existe de nombreuses solutions permettant de contrôler el de commander à distance une installation de quelque importance qu'elle soit. Nous allons en examiner sommairement quelques-unes parmi les plus couramment employées.

\section{'Télécommantes par fils pilotes et relais sensibles}

On utilise généralement, pour les canalisations reliant l'installation au poste de commande, des câbles muliipaires de conducteurs de la « série téléphonique ». Ces câbles comportent un certain nombre de conducteurs isolés au papier, placés sous chemise plomb. Les conducteurs sont en cuivre et leur diamètre varie habituellement, selon la distance, entre 0,5 et $1,2 \mathrm{~mm}$. pour atteindre parfois 1,5 et même $2 \mathrm{~mm}$. Certains câbles de la série « téléphonique » peuvent renfermer jusqu'à plusieurs centaines de paires de conducteurs. Si les câbles sont installés en «aérien », on les suspend à un fil d'acier accroché aux supports d'une ligne existante par le système de suspension «caténaire » couramment utilisé par l'administration des P. T. T. S'ils sont placés en «tranchée » il faut utiliser des câbles protégés en outre par filins avec armature en feuillards d'acier.

La source auxiliaire alimentant le dispositif est habituellement constituée par une batterie de piles, ou de préférence d'accumulateurs de quelques dizaines de volts seulcment, les câbles de la série téléphonique n'étant pas suscepitibles de supporter sans danger des tensions élevées. Si les distances conduisent à utiliser des tensions plus grandes (de l'ordre de $110 / 120$ volts par exemple), les cábles doivent être établis avec isolement renforcé capable de supporter sans inconvénient de telles tensions.

Pour les distances relativement importantes ou pour les canalisations pilotes longeant une ligne électrique à hautetension, il n'est pas recommandé d'utiliser des lignes aériennes à conducteurs nus, les phénomènes d'induction dus à la proximité des conducteurs haute-tension ou aux décharges atmosphériques étant susceptibles d'entraîner des avaries ou des perturbations sérieuses dans le fonctionnement du dispositif.

L'emploi du courant continu permet d'utiliser des relais très sensibles et des relais polarisés réduisant au minimum le nombre de fils pilotes reliant l'installation au poste de commande. En principe il faut compter un fil par appareil

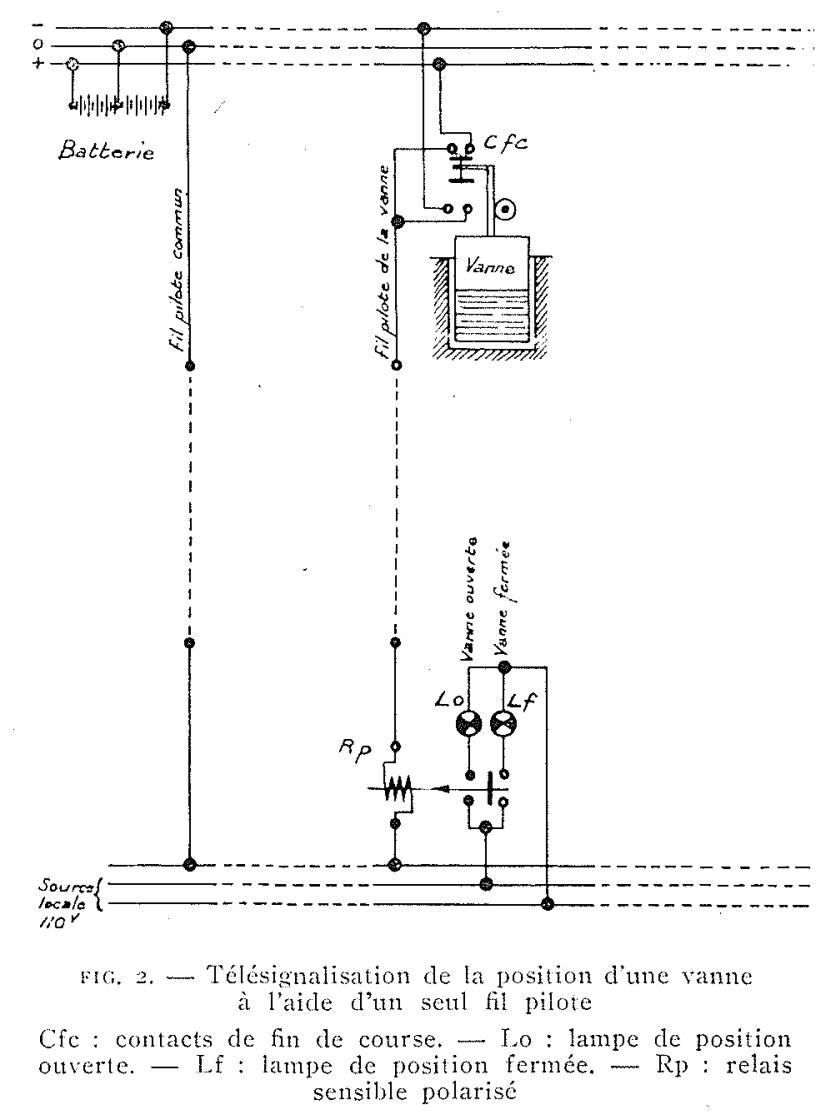

signalé et commandé à distance, plus deux ou trois fils communs à l'ensemble de l'installation. A ce chiffre viennent s'ajouter les conducteurs supplémentaires destinés aux télémesures.

Le schéma de la figure $n$ " 2 indique, à titre d'exemple, un dispositif de télésignalisation pour la position d'une vanne. Deux lampes Lo et $L f$, situées au poste de commande, indiquent si la vanne est ouverte ou fermée. Pendant toute la durée de la manœuvre les 2 lampes sont éteintes. Il en est de même si la vanne s'arrête, pour une raison quelconque, entre ses deux positions extrêmes.

La figure $n^{\circ} 3$ donne un exemple de schéma pour la signalisation et la tólécommande d'un disjoncteur à l'aide d'un «Turn-Push » inclus dans un schéma unifilaire figurant l'installation. Le turn-push possède une lampe centrale unique, normalement éteinte lorsque la position de la barrette dans le schéma coincide avec la position du disjoncteur signalé.

Lorsqu'on veut enclencher ou déclencher à distance le disjoncteur, on tourne préalablement la barrette du turnpush. Le relais de haute sensibilité RHS est mis sous tension, la lampe centrale s'allume et la sonnerie retentit. Il est à noter que le courant qui traverse le circuit pilote à ce moment est très inférieur à celui nécessaire pour actionner le relais polarisé RP qui reste au repos. On 


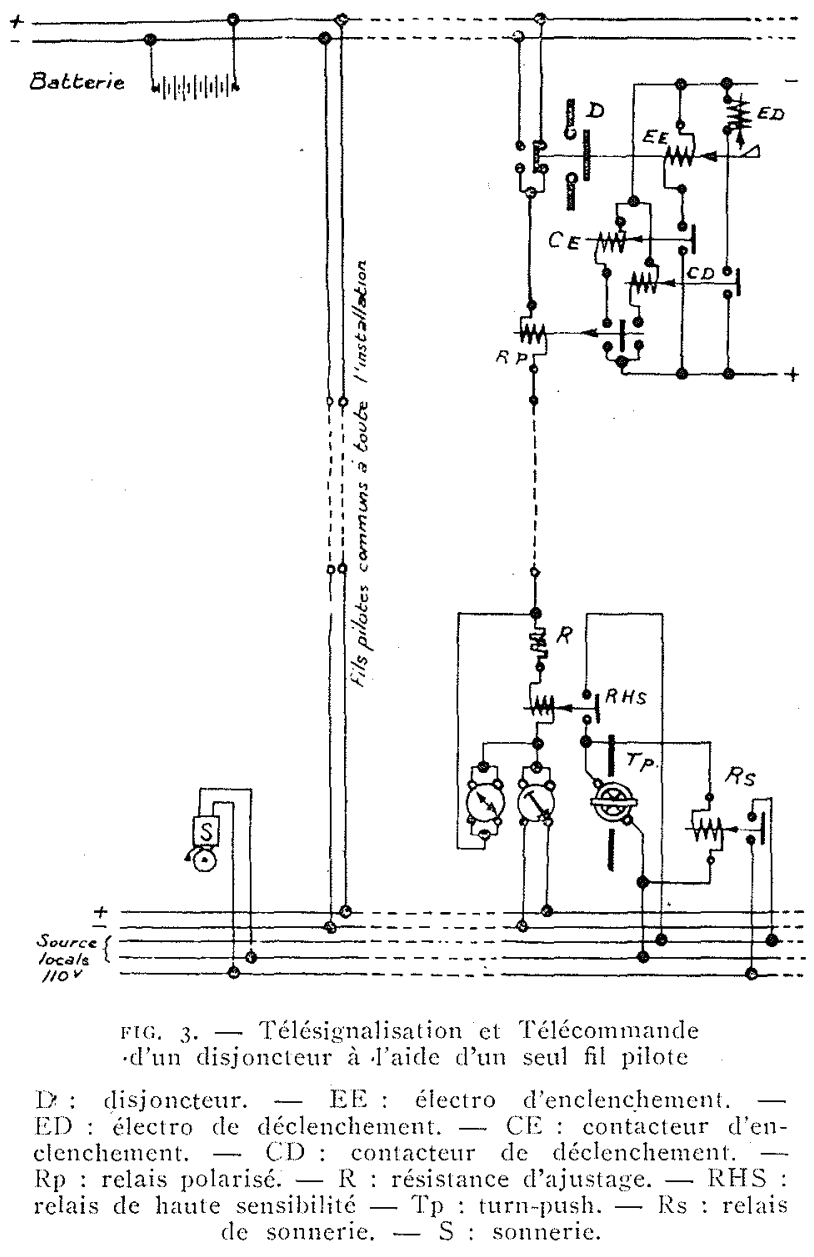

presse alors sur la barrette du turn-push, ce qui a pour effet de court-circuiter le relais RHS et la résistance d'ajustage. Le relais polarisé RP est alors mis sous pleine tension et, selon le sens du courant qui traverse son bobinage, sens déterminé par la position initiale du disjoncteur, il provoque la manœuvre désirée.

Les contacts auxiliaires du disjoncteur changent la polarité initiale au départ du fil pilote; le relais RHS tombe au repos, la lampe s'éteint et la sonnerie s'arrête, indiquant à l'opérateur que la manœuvre s'est correctement effectuée.

$\mathrm{Si}$ le disjoncteur déclenche automatiquement sous l'action de ses relais de protection, la polarité au départ du fil pilote est inversée, la lampe s'allume et la sonnerie retentit. L'électricien de service au poste de commande peut alors, soit provoquer le réenclenchement en pressant sur la barrette comme indiqué plus haut, soit entériner la nouvelle position du disjoncteur en tournant la barrette pour la placer dans la nouvelle position correspondante, ce qui a pour effet d'inverser la polarité à l'arrivée du fil pilote. Le relais RHS tombe au repos, la lampe s'éteint et la sonnerie s'arrêté.

\section{Télécommandes par fils pilotes at melais sélecteurs}

Ce système de télécommande comporte un ensemble de dispositifs semblables à ceux utilisés pour la téléphonie automatique. La ligne assurant la liaison entre le poste de commande et l'installation télécommandée peut ne comporter que deux conducteurs.

Comme pour le dispositif précédemment décrit, des précautions doivent être prises pour éviter toutes perturbations ou détériorations pouvant résulter des phénomènes d'induction sur les fils pilotes, lorsque ceux-ci sont placés eil « aérien ».

Des relais sélecteurs dont l'avancement est synchronisé avec les impulsions lancées depuis le poste de commande «cherchent » le circuit correspondant à l'appareil dont on veut connaître la position exacte ou que l'on veut manœuvrer. Des dispositifs spéciaux sont prévus pour éviter toutes possibilités de fausses manouvres pour le cas où, par suite d'un dérangement quelconque, le sélecteur s'arrêterait sur une position différente de celle commandée par le système émetteur d'impulsions.

Une fois le circuit établi, des impulsions de commande

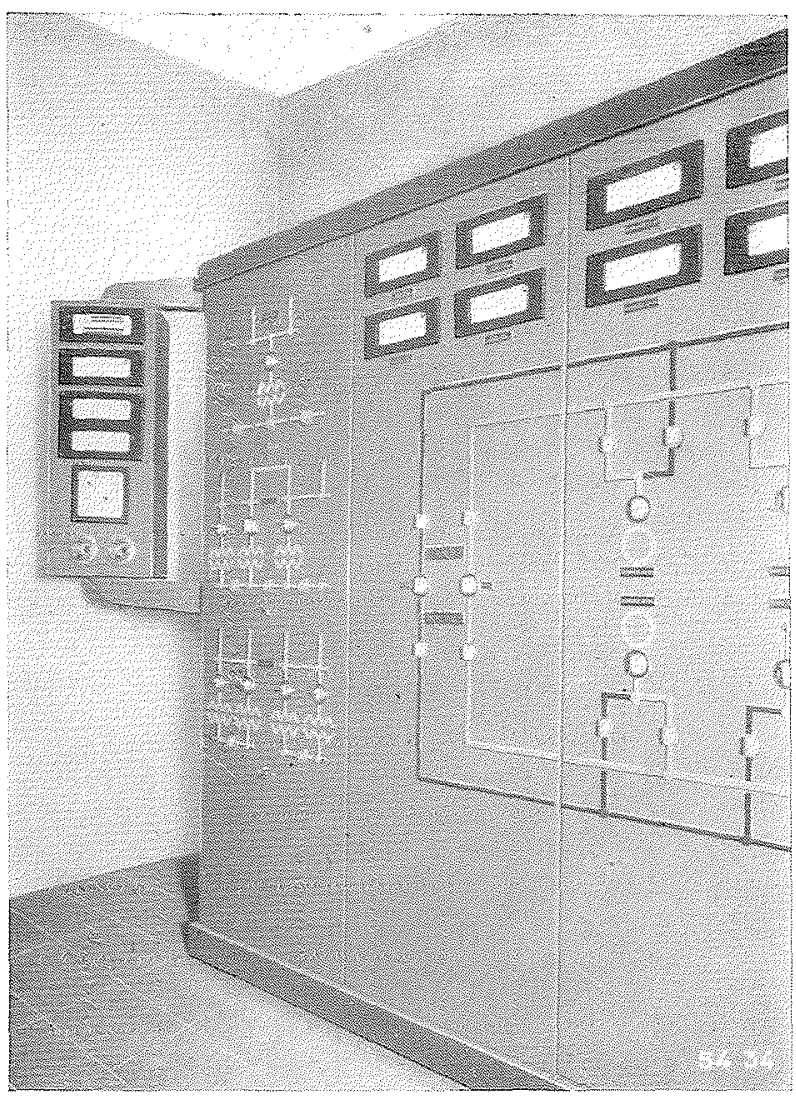

TABLEAU DE COMMANDE AVEC SCHÉMA SYNOPTIQUE ÉQUIPÉ AVEC 《TURN-PUSH 》 


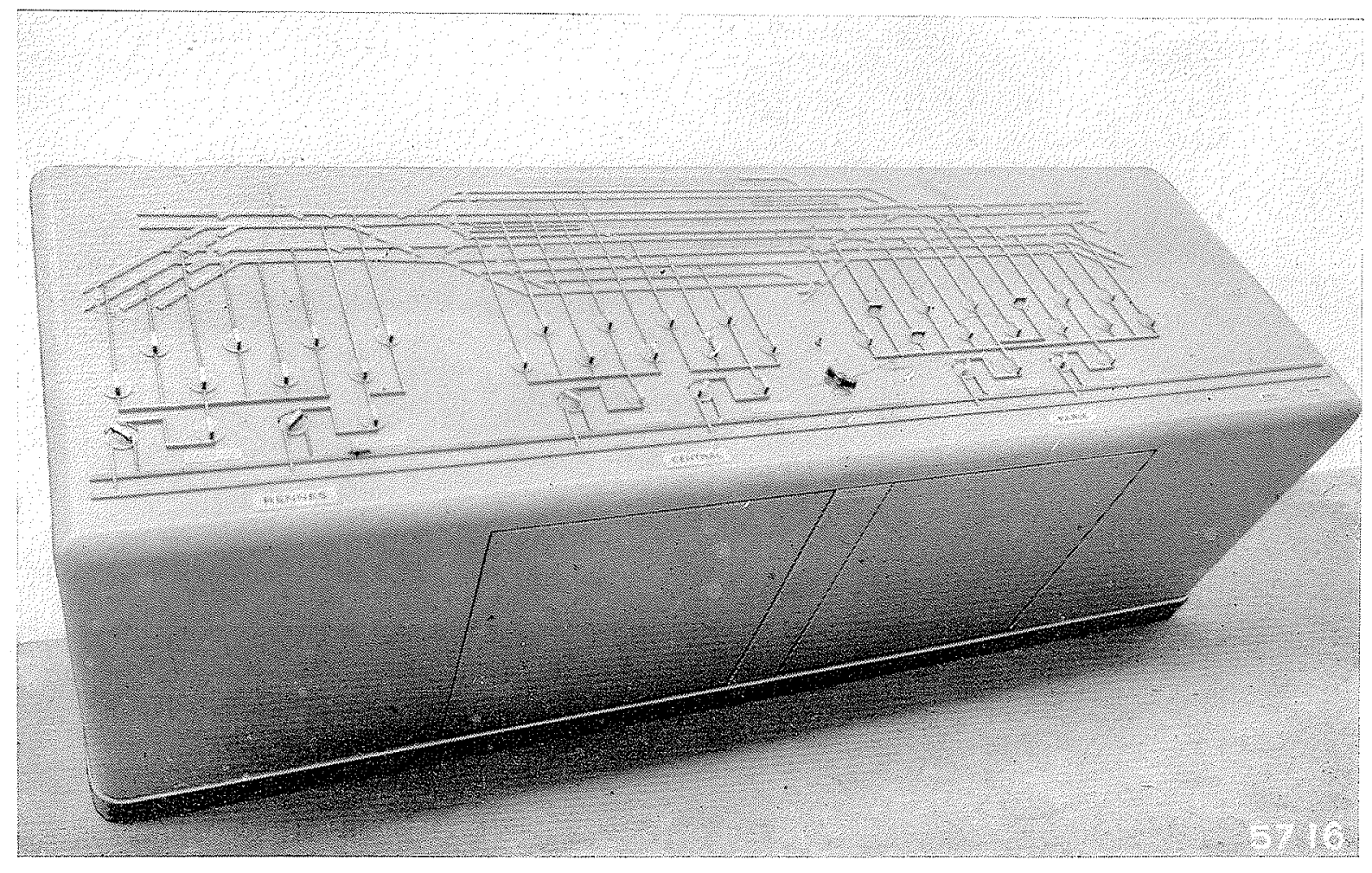

PUPITRE DE COMMANDE ÉQUIPÉ AYEC «TURN-PUSH 》

sont lancées pour modifier la position de l'appareil que l'on veut manceuvrer. En retour, les appareils indicateurs de position du poste de commande reçoivent, depuis les contacts auxiliaires placés sur l'appareil que l'on vient de manæuvrer, les impulsions destinées à rectifier leurs indications, ce qui confrrme à l'opérateur que la manœuvre désirée s'est effectuée correctement.

Lorsque la position d'un appareil est modifiée sans intervention de la télécommande (c'est le cas par exemple du déclencheur automatique d'un disjoncteur) le dispositif se met immédiatement en route pour aviser le poste central de commande de la modification intervenue à son insu.

Aucune liaison électrique permanente n'existant entre les appareils de l'installation télécommandśe et leur signalisation au poste de commande, les relais assurant la répétition des signaux dans ce dernier poste conservent la position imposée par les dernières impulsions reçues jusqu’à ce qu'ils soient influencés par de nouvelles impulsions. L'électricien de service au poste de commande peut obtenir une vérification générale de l'exactitude de toutes les signalisations en provoquant un «balayage» successif, par les sélecteurs, des circuits de signalisation de tous les appareils de l'installation télécommandée.

\section{Télécommandes par fils pilotes el chaines de relais a fonctionnement synchrone}

Ce dispositif se rapproche sensiblement de celui décrit précédemment mais les relais sélecteurs sont remplacés par une double chaîne de relais ordinaires, la première chaîne étant situce au poste d'émission, la seconde au poste récepteur. Il nécessite en général 4 fils pilotes.

Supposons que l'on veuille manœuvrer à distance un disjoncteur qui occupe le 4 rang dans l'ordre de préséance des circuits de signalisation et de commande de tous les appareils de l'installation susceptibles d'être signalés ou manœurrés à distance, et que la commande soit efiectuée à l'aide d'un turn-push semblable à celui décrit dans l'un des dispositifs précédents.

En plaçant la barrette de ce turn-push sur la position désirée, on provoque au poste d'émission la fermeture du relais de rang 1 de la première chaîne; ce relais provoque à son tour la fermeture clu relais de rang 1 de la deuxième chaine qui, en retour, provoque la fermeture du relais de rang 2 de la première chaîne. Le relais de rang 2 de la première chaîne provoque la fermeture du relais de rang 2 de la deuxième chaîne qui, en retour, entraîne 
la fermeture du relais de rang 3 de la première chaîne, et ainsi de suite jusqu'à ce que les deux relais de rang 4 des deux chaînes soient fermés simultanément à chaque extrémité de la ligne.

Le fonctionnement en «cascade 》 des relais' des deux chaînes, qui s'effectue à une cadence extrêmement rapide, s'arrête alors automatiquement. On peut à ce moment envoyer l'impulsion de commande et on reçoit, en retour, l'indication que la manœuvre s'est effectuée correctement.

\section{Télécommandes par émissions à fréquences musicales avec ou sans fils pilotes}

De tels systèmes s'appliquent à la commande et à la signalisation en retour d'un nombre quelconque d'appareils répartis dans un ou plusieurs postes qui peuvent être très éloignés du poste de commande (quelques centaines de kilomètres), pourvu qu'ils soient reliés à ce dernier par un même canal de transmission.

Chaque ordre à transmettre ou signalisation à recevoir est caractérisé par l'émission d'un ou de plusieurs groupes successifs de courants à fréquences musicales. Le nombre de combinaisons ainsi obtenues étant très élevé, il devient alors possible de commander et de contrôler à distance, si besoin est, plusieurs milliers d'appareils répartis sur l'ensemble d'un réseau.

Ces systèmes, spécialement étudiés pour être insensibles aux influences parasites, peuvent utiliser entre le poste de commande et les installations commandées, soit une ligne téléphonique ordinaire à 2 ou à 4 conducteurs comportant ou non des répéteurs, soit un courant porteur émis directement sur les conducteurs de lignes à haute-tension, ce qui supprime totalement l'installation de fils pilotes ou de lignes téléphoniques.

Une installation de ce genre comporte, au poste de commande, un ensemble émetteur et un ensemble récepteur de courants à fréquences musicales, avec les dispositifs amplificateurs nécessaires et les relais auxiliaires pour démarrage des émissions et pour transmission des signalisations reçues aux appareils indicateurs.

Chacun des postes commandés comporte également un ensemble récepteur et un ensemble émetteur de courants à fréquences musicales, avec leurs dispositifs amplifica-

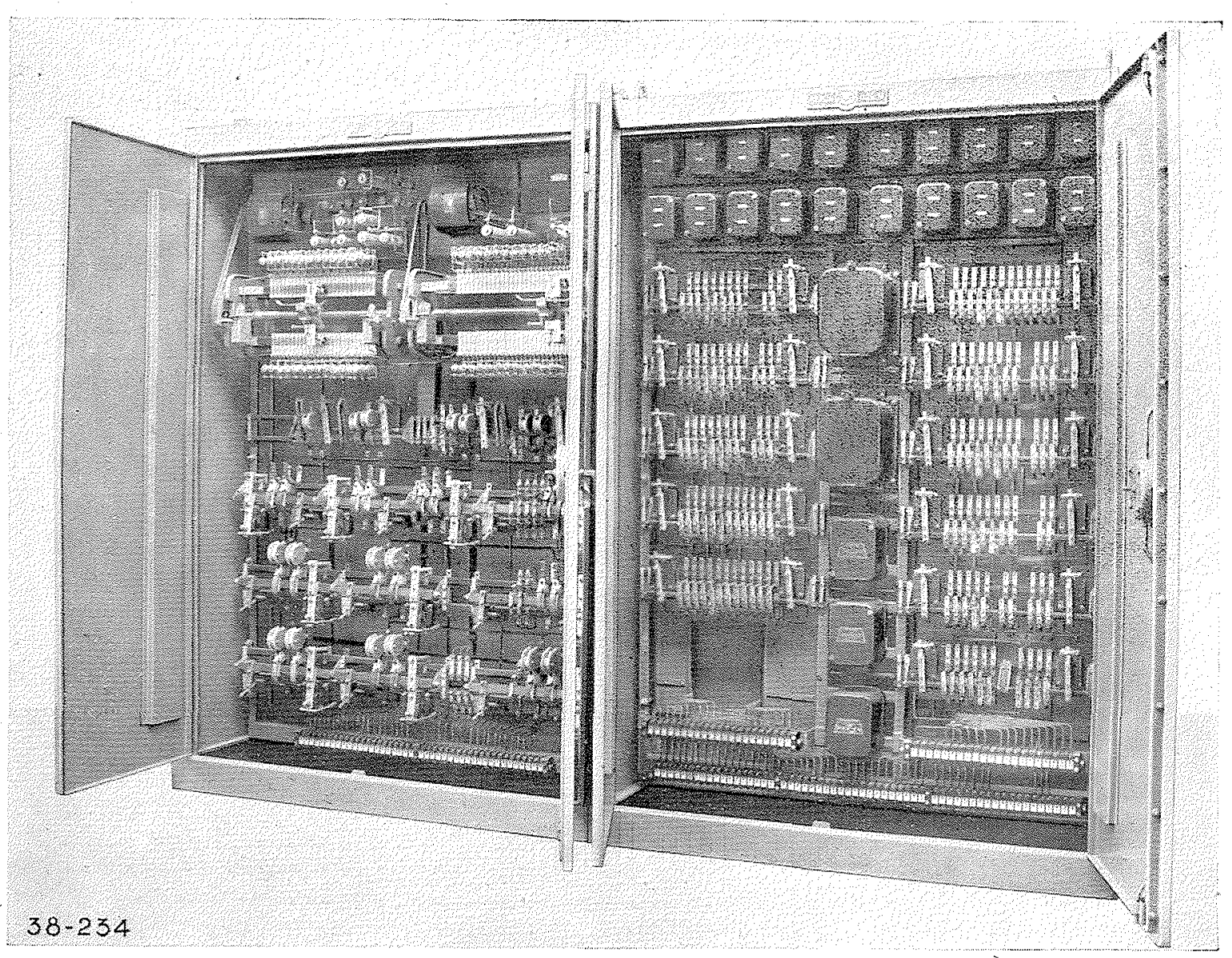

ARMOIRE POUR DISPOSITIF DE COMMANDE A DISTANCE

ÉQUIPÉE AVEC RELAIS A FRÉQUENCE MUSICALE SYSTÈME «ACTADIS 》 
teurs et les relais auxiliaires pour réception des ordres de manœuvre et démarrage des émissions de signalisation.

Dans chacun des postes de commande et de réception, l'énergie nécessaire est empruntée, comme pour les systèmes précédemment décrits, aux sources auxiliaires locales.

Tout déclenchement automatique d'un disjoncteur ou toute modification à la position d'un appareil non provoquée par le canal de la télécommande, entraînent automatiquement les émissions destinées à signaler, au poste central de commande, les modifications intervenues.

\section{TÉLEMESURES}

Comme indiqué au début de la présente étude, les dispositifs de signalisation et de commande à distance doivent souvent être complétés par la transmission au poste de commande des mesures essentielles, telles que tension, intensité, puissance, etc...

Lorsque la distance est peu importante, les mesures simples de tension ou d'intensité en courant alternatif peuvent être transmises avec une précision suffisante sous forme de courant continu de quelques milliampères recueillis aux bornes de petits redresseurs secs, spécialement ćtablis dans ce but, et alimentés par des transformateurs réducteurs directement branchés aux bornes secondaires des transformateurs de tension ou d'intensité de l'installation. Les appareils récepteurs sont des galvanomètres ordinaires à cadre mobile, fictivement gradués en volts ou en ampères. Leur échelle doit évidemment correspondre au calibre nominal du primaire du transformateur de mesure placé à l'origine de leur alimentation.

Pour des distances plus importantes, ou pour des mesures complexes telles que des mesures de puissance par exemple, on utilise des appareils transmetteurs spéciaux actionnant à distance les appareils récepteurs convenablement gradués.

Les transmissions sous forme de courant continu proportionnel nécessitent des fils pilotes supplémentaires qui peuvent être inclus dans les câbles à conducteurs multiples uitilisés pour les tálésignalisations et les télécommandes. Le nombre de conducteurs supplémentaires est directement proportionnel au nombre de mesures à transmettre.

Les transmissions peuvent également s'effectuer sous forme d'émissions à haute fréquence.

Si l'on doit transmettre plusieurs mesures sur des pilotes communs, il devient nécessaire d'utiliser des systèmes à impulsions transmises par l'intermédiaire de relais sélecteurs synchronisés, ou dispositifs analogues semblables à ceux décrits plus haut pour les télécommandes. Les appareils de mesures récepteurs sont alors d'un modèle spécial, dont l'équipage mobile reste dans la position où l'a placé la dernière impulsion reçue, jusqu'à ce qu'il soit influencé par une nouvelle émission.

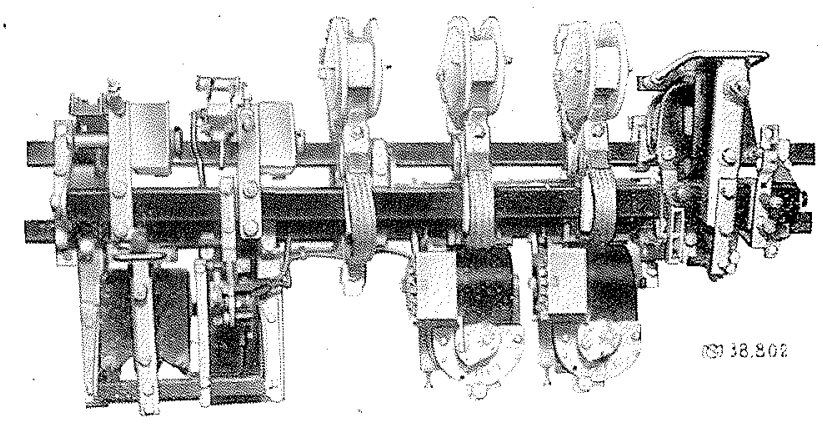

Contacteur basse tension arec disposilif de réenclenchanents multiples et automatiques

\section{INSTALLATIONS AUTOMATIQUES}

Il ne peut être question ici de décrire tous les cas d'installations de ce genre susceptibles de se présenter.

Parmi les installations les plus courantes, nous ne rappellerons que pour mémoire les équipements de démarrage en plusieurs temps des moteurs asynchrones ou synchrones alimentés directement en haute-tension, et les équipements basse-tension à contacteurs assurant la mise en marche ou l'arrêt automatique de certaines machines, telles que les moto-pompes, les compresseurs, etc..., en fonction, par exemple, du niveau d'un liquide ou d'une pression dans un réservoir. De telles installations sont en-

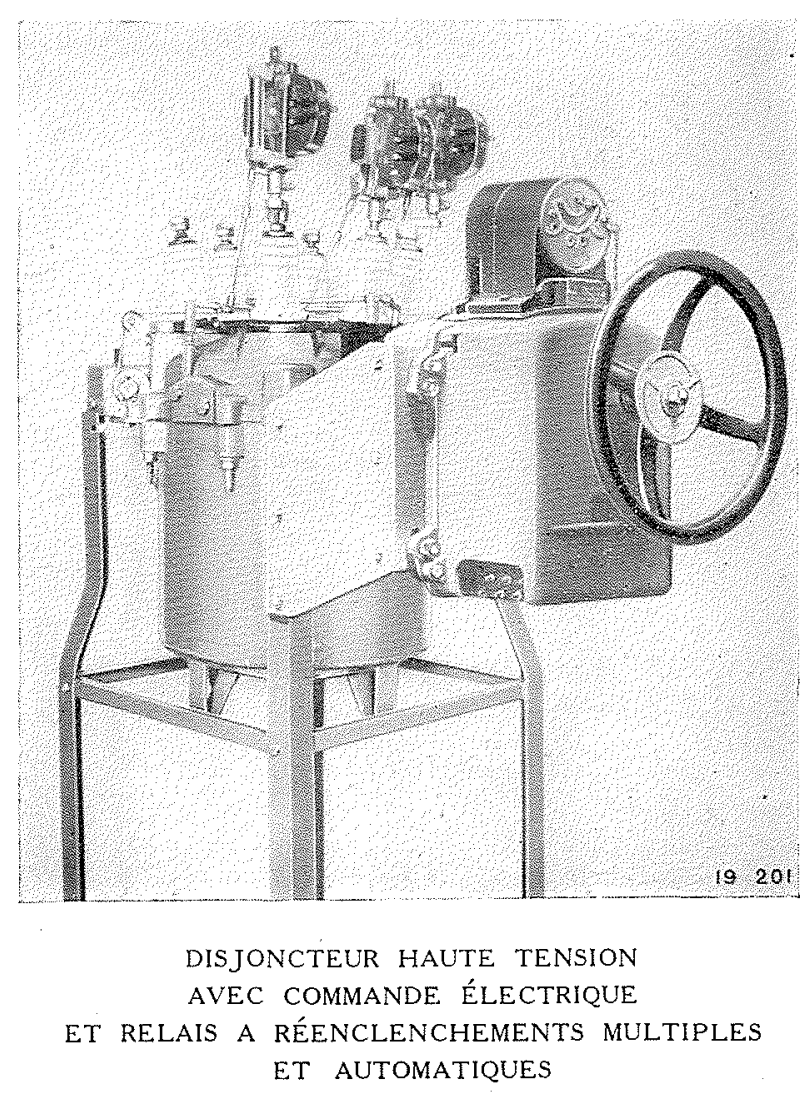


trées dans la pratique courante et sont universellement connues.

Dans un poste de distribution d'énergie, l'installation a.utomatique la plus simple est sans conteste le disjoncteur équipé avec relais à réenclenchements multiples, qui provoque sans aucune intervention humaine un certain nombre de tentatives de remise en service d'un feeder à des intervalles de temps déterminés, avant de laisser finalement l'appareil dans la position ouverte si le défaut ayant provoqué la disjonction persiste. Certains relais sont placés directement sur la commande de l'appareil et agissent mécaniquement ou électriquement sur cette dernière. D'autres se présentent en boîtier indśpendant et peuvent s'installer avec d'autres appareils sur un tableau $d z$ commande.

Des installations, très simples également, provoquent la mise en service automatique d'un ou de plusieurs circuits à certaines heures de la journée ou de la nuit. Elles sont commandées à l'origine par une horloge mère du modèle courant, agissant sur les commandes électriques des disjoncteurs haute ou basse-tension par l'intermédiaire de relais ou contacteurs du modèle approprié. Des dispositifs de ce genre sont notamment utilisés pour le chauffage de chaudières électriques à accumulation pendant les périodes creuses de la distribution d'énergie.

Nous examinerons plus particulièrement ici quelques cas «types»d'installations automatiques plus complexes.

\section{Postes répartiteu's automatiques}

Voici à titre d'exemple une description sommaire d'une installation de ce genre.

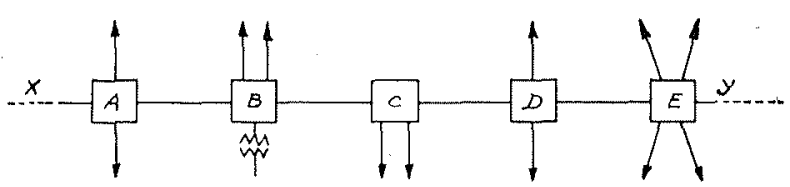

Fla, 4. - Série de postes répartiteurs intercalés sur une lipne à haute tension alimentée à ses cletx extrémités

Considérons une série de postes répartiteurs $A, B, C, D$, intercalés sur une ligne haute-tension $X Y$ d'un réseau bouclé suivant schéma de la figure $n^{\circ} 4$, cette ligne étant normalement alimentée par ses deux extrémités.

La figure $\mathrm{n}^{\circ} 5$ donne le schéma de l'un de ces postes, le poste $C$ par exemple; sur ce schéma sont simplement figurés les appareils essentiels pour le fonctionnement du dispositif, notamment les trois disjoncteurs $D_{1}, D_{2}, D_{i}$ et le sectionneur inverseur I. Ces appareils sont tous munis d'une commande électrique dont l'alimentation est assurée par les transformateurs $\mathrm{Tp}_{\mathrm{p}-1}$ et $\mathrm{Tp}$-2 $_{2}$ ou par une batterie d'accumulateurs auxiliaires.

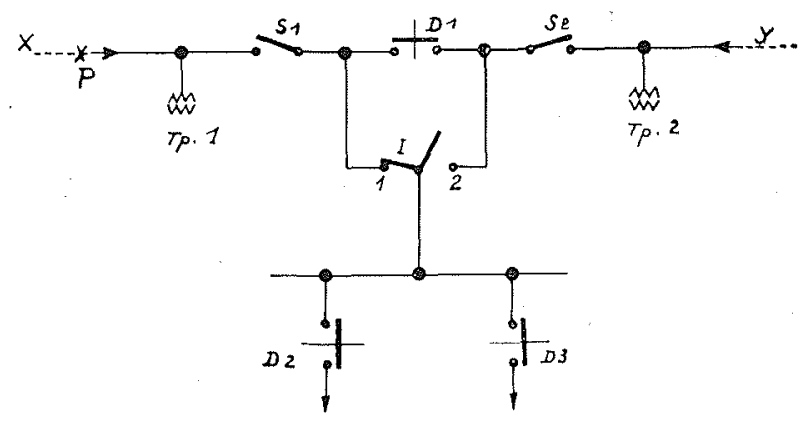

rá. 5. - Schéma mifiaire hate tension du poste C de la fisure precédente

En péricde normale d'exploitation le réseau est bouclé, tous les appareils figurés sur le schéma sont fermés, l'inverseur étant par exemple sur la position 1 .

Les disjoncteurs de ligne $D_{1}$ de tous les postes sont munis d'une pootection à maxima d'intensité affectée de deux tcmporisations distinctes, l'une ou l'autre de ces temporisations étant mise en service par un relais directionnel selon le sens d'écoulement du courant de défaut. Pour chaque sens considéré, ces temporisations sont réglées d'autant plus élevées que l'on se rapproche des sources d'énergie, afin d'obtenir une sélection convenable et ds n'éliminer que le tronçon de ligne sur lequel un défaut s'est produit. Un relais à réenclenchements multiples assure sous certaines conditions seulement le réenclenchement do ce disjoncteur, un verrouillage approprié interdisant notamment ce rćenclenchement si les deux côtós de la ligne sont sous tension.

Les disjoncteurs $D_{3}$ et $D_{3}$ sont munis d'une protection à maxima d'intensité temporisée, d'un relais à minimum de tensicn retardá et d'un relais à réenclenchements multiples.

Supposons qu'un défaut se produise au point $P$, sur la ligne reliant les postes $B$ et $C$. Les deux disjoncteurs des postes $B$ et $C$ déclenchent au bout de leur temporisation respective, selon le sens de circulation du défaut. Les relais à réenclenchements multiples sont mis en service, toutefois, d’̀s que l'un des disjoncteurs a effectué un réenclenchement, les deux arrivées de la ligne aboutissant à l'autre disjoncteur sont sous tension, ce qui interdit 1) réenclenchement de l'autre disjoncteur.

Si le défaut persiste, le disjoncteur ayant réenclenché déclenche à nouveau et se bloque dans cette position lorsque le relais à réenclenchements aura effectué le nombre dét tentatives (réglable à volonté) qui lui aura été assigné. Le tronçon de ligne accidenté est alors défnitivement mis hors de service.

Les départs $D_{2}$ et $D_{3}$, qui étaient initialement raccordés au tronçon accidenté, ne sont plus alimentés. Le dispositif d'automaticité qui leur est propre intervient alors pour les commuter sur l'arrivée restée sous tension. Le 
sectionneur I ne pouvant être manœuvré en charge, le cycle de manouvre est le suivant :

10 Déclenchement des disjoncteurs $D_{2}$ et $D_{33}$. Ce déclenchement se produit sous l'action des relais à minima de tension au bout de la temporisation assignée à ces relais. Cette temporisation a pour but d'interdire toute manœuvre de commutation tant que le disjoncteur $D_{\text {, }}$ effectue lui-même des tentatives de remise sous tension.

2" Passage de l'inverseur I de la position 1 à la position 2. Cette mancuvre est effectuée par la commande électrique de l'inverseur. Le circuit d'asservissement do cette commande électrique passe par des contacts en série montés respectivement sur les disjoncteurs $D_{\underline{\text { In }}}$ et $\mathrm{D}_{3 ;}$ et fermés lorsque les disjoncteurs sont ouverts. La commande électrique ne peut donc être actionnée que si tous les départs sont déclenchés, ce qui donne la certitude que l'inverseur ne pourra manæuvrer en charge.

$3^{\prime}$ Réenclenchement des disjoncteurs $D_{2}$ et $D_{33}$. Cette manœuvre est commandée par les relais à réenclenchements multiples affectés à ces deux appareils au bout de la temporisation choisie pour ces relais. En outre, des contacts de sécurité placés sur l'inverseur I et intercalés sur le circuit de commande des relais à réenclenchements multiples interdisent tout réenclenchement intempestif pendant le fonctionnement de l'inverseur.

Les divers tronçons de l'instạllation sont donc à nouveau en service à l'exclusion du tronçon accidenté qui a été éliminé. Le poste répartiteur a joué automatiquement son rôle sans aucune intervention humaine.

\section{Postes de transformation antomatiques}

Certains postes de transformation alimentent des installations dont la consommation d'énergie est extrêmement variable dans le temps. Ceci se produit notamment pour l'alimentation de certaines usines comportant des fours et pour certains secteurs ruraux. La nécessité d'assurer l'alimentation sans défaillance conduit à laisser en service, en permanence, des transformateurs dont la puissance spécifique est très nettement plus élevée que la puissance moyenne appelée par le réseau. II en résulte un mauvais rendement de l'installation du fait que les pertes à vide du transformateur sont élevées et le facteur de puissance relativement bas aux faibles charges.

Une solution plus rationnelle consiste à prévoir plusieurs transformateurs dont la somme des puissances spécifiques est suffisante pour répondre à la puissance maximum du réseau, et à ne mettre en scrvice que le nombre d'unités strictement nécessaires pour répondre à la puissance du moment. Le dispositif d'automaticité intervient alors pour mettre «en》 ou « hors 》 service chaque transformateur suivant un ordre de préséance prédéterminé, et ceci en fonction des fluctuations de l'appel de courant du réseau.
Un tel dispositif comporte, sur l'arrivée du courant à haute-tension, un système détecteur constitué par autant de groupes de relais qu'il y a de transformateurs à commander automatiquement dans le poste. Ces relais sont alimentés par des transformateurs d'intensité haute-tension. Ils se ferment lorsque le courant appelé par l'installation nécessite la mise en service du transformateur auquel ils sont affectés, et s'ouvrent lorsque ce transformateur devient inutile.

Les contacts de ces relais actionnent des relais auxiliaires à contacts temporisés qui agissent directement sur la commande électrique des disjoncteurs haute-tension protégeant les transformateurs pour en provoquer l'enclenchement ou le déclenchement. Sur le côté basse-tension de ces mêmes transformateurs sont généralement prévus de simples contacteurs commandés par un contact auxiliaire fermé, disjoncteur fermé, monté sur le disjoncteur H.T., de telle sorte que le contacteur se ferme lorsque le disjoncteur H.T. s'enclenche, et s'ouvre lorsque le disjoncteur déclenche.

La temporisation des contacts de chaque relais auxiliaire est choisie de telle sorte qu'une pointe passagère de courant telle que celle due au démarrage d'un moteur par exemple, ou une baisse momentanée d'intensité n'excédant pas quelques secondes, ne puissent provoquer le fonctionnement du système.

Un circuit de sécurité est en outre prévu pour chaque transformateur en cas de dérangement du dispositif. II peut, au choix, soit actionner un avertisseur, soit provoquer la substitution automatique d'une autre unité à l'unité défaillante, soit même provoquer le déclenchement du disjoncteur général. On a ainsi la certitude de ne pas surcharger longtemps d'une manière dangereuse les unités en service, si, pour une raison quelconque, la mise en service de l'un des transformateurs ne s'est pas effectuée correctement.

Des commutateurs appropriés permettent de mettre à volonté le dispositif de commande automatique «en» ou «hors》 service, et de modifier l'ordre de préséance des divers transformateurs du poste pour placer au dernier stade une unité douteuse ou éliminer momentanément une unité avariée sans interrompre la continuité de fonctionnement du système.

Le fonctionnement des dispositifs de protection interne des transformateurs, tels que relais Buccholz, thermomètres à contact, relais différentiels, relais de mise à la masse, etc..., doit entraîner l'élimination du transformateur avarié provoquant le déclenchement immédiat du disjoncteur et du contacteur qui l'encadrent. Les unités restáes en service vont de ce fait se trouver surchargées, et les dispositifs de sécurité doivent intervenir comme indiqué plus haut en actionnant un avertisseur, en provoquant la substitution d'une autre unité à celle qui est avariée ou le déclenchement du disjoncteur général. Il faut tenir compte 
à ce sujet qu'avec des transformateurs modernes de bonne construction, ce genre d'incident est extrêmement peu fréquent.

Des précautions toutes spéciales doivent être prises en ce qui concerne le choix du système de protection à maxima d'intensité des transformateurs. Les relais à maxima d'intensité choisis doivent être munis d'une temporisation constante, et cette temporisation doit être suffisante pour permettre au dispositif d'automaticité de coupler un transformateur supplémentaire avant de faire déclencher les unités déchargées. Une bonne solution consiste à utiliser les relais du système détecteur eux-mêmes par l'intermédiaire des circuits de sécurité. On obtient ainsi une protection à maxima qui est toujours en harmonie avec le nombre d'unités en service, mais ceci implique qu'en courant triphasé par exemple, chaque groupe détecteur comprenne au moins deux relais branchés sur deux phases distinctes pour que toute surcharge n'intéressant qu'une seule phase soit détectée par ce groupe de relais.

La figure $\mathrm{n}^{\circ} 6$ donne le schéma d'une installation automatique de ce genre dans laquelle les couplages de transformateurs se font par tranches de $200 \mathrm{kva}$. Remarquer que le poste ne comporte qu'un seul transformateur de cette puissance qui se met «en 》 ou « hors » service entre chaque insertion ou suppression d'une unité de 400 kva.

\section{Centrales automatiques ou semi-automatiques}

Une centrale semi-automatique diffère d'une centrale automatique en ce sens que seules certaines manœuvres seffectuent sans aucune intervention humaine. Par exemple, la mise en route ou l'arrêt peuvent être télécommandés à l'origine depuis une installation voisine ou un poste central de commande. Les opérations nécessaires de mise en vitesse, d'excitation, d'amenée au synchronisme et de couplage s'effectuent ensuite automatiquement. Une fois ce stade atteint, la régulation de la charge peut être télécommandée.

Par contre, dans la centrale entièrement automatique, une machine' se met en route, règle sa charge et s'arrête d'elle-même suivant un programme fixé à l'avance.

Ce programme est généralement basé sur les considérations suivantes :

quantité d'eau disponible au barrage, quantité d'eau à débiter au canal de fuite, maintien d'un niveau d'eau constant au barrage,

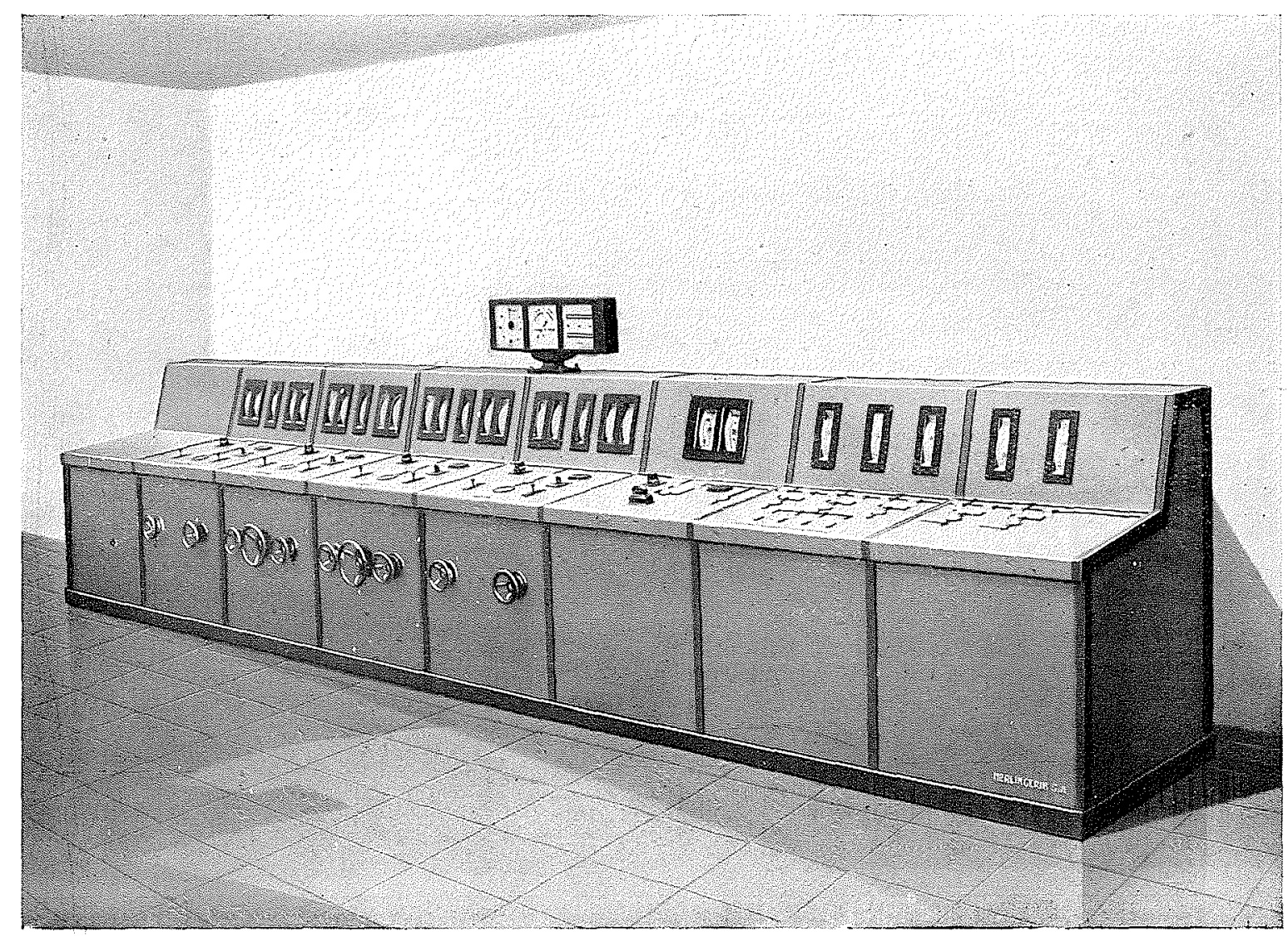

PUPITRE DE COMMANDE ET DE CONTROLE AVEC SCHÉMA SYMBOLIQUE TYPE « OBSCUR» 
maintien d'un niveau constant dans le canal de fuite quel que soit le débit de ce canal (cas des irrigations),

fourniture des appoints d'énergie nécessaires au passage des heures de pointe,

respect d'un programme journalier résultant des contrats de fourniture d'énergie,

etc..., etc...

A l'origine du dispositif de commande automatique se trouvent, soit des flotteurs munis de contacts auxiliaires, soit une horloge mère, soit un dispositif wattmétrique, soit tout autre système spécialement conçu pour provoquer la mise en route ou l'arrêt des machines dans des conditions bien définies.

Les diverses opérations de mise en service d'une machine forment un cycle de manœuvres. Celles-ci s'effectuent dans un ordre prédéterminé.

La première impulsion de commande met habituellement en route la pompe électrique de mise en pression du régulateur et éventuellement une pompe de graissage. Dès que la pression pour ces deux fonctions atteint une valeur suffisante, des contacts manométriques provoquent l'ouverture de la vanne d'admission. En fin de course de cette vanne, un contact agit sur le régulateur de la machine. La turbine démarre et accélère sa vitesse dont le contrôle est alors assuré par le régulateur lui-même. Au voisinage de la vitesse de synchronisme, un contact tachymétrique ou un relais actionné par une dynamo tachymétrique montée en bout d'arbre, provoque la fermeture du circuit d'excitation et la mise en service des appareils de couplage.

Le régulateur de tension de la machine intervient alors pour régler l'excitation à une valeur convenable. Les pompes mécaniques sont entraînées à leur vitesse normale et les pompes électriques s'arrêtent.

Le synchroniseur automatique en agissant sur le régulateur provoquc des variations de vitesse dans le but d'amener celle-ci au synchronisme. Lorsque la concordance de phase est atteinte avec une précision suffisante, le coupleur automatique provoque la fermeture du disjoncteur haute-tension. La machine est couplée sur le réseau.

Des contacts auxiliaires placés sur le disjoncteur hautetension libèrent les appareils de couplage qui tombent au repos. D'autres contacts placent le régulateur sous la dépendance du dispositif de contrôle cle la charge (flotteur, 'système wattmétrique ou autre) qui agit sur le régulateur de la turbine dans le sens ouverture jusqu'à ce que la puissance atteigne le chiffre réalisunt le programme prévu. Le régulateur de tension ajuste parallè. lement le courant d'excitation à une valeur convenable.

A partir de ce moment, la machine est normalement en service. Elle suivra fidelement les fluctuations de puissance qui lui sont imposées par le dispositif de contrôle précité.
Si l'asservissement se fait par rapport au niveau d'eau dans le canal de fuite, il se peut que le flotteur agisse directement et mécaniquement sur le régulateur de la turbine si les conditions locales d'installation le permettent; mais le plus souvent ces flotteurs agissent électriquement sur le moteur de commande du régulateur par l'intermédiaire de relais auxiliaires.

Dans le cas particulier ou la puissance débitée doit être constante dans le temps, on prévoit des relais ampèremétriques ou wattmétriques à maximum et à minimum qui agissent électriquement dans le sens convenable sur le moteur de commande du régulateur.

Si cette puissance doit être variable dans le temps (c'est-à-dire réglée à des valeurs différentes à certaines heures de la journée), les relais précités peuvent être combinés avec un dispositif actionné par une horloge mère qui modifie leur sensibilité en agissant par exemple sur le rapport des transformateurs d'intensité alimentant les relais ampèremétriques ou wattmétriques sus-indiqués.

Enfin, si la puissance débitée doit suivre un programme journalier susceptible d'être modifié assez fréquemment, on peut utiliser un régulateur de puissance spécial assez semblable à un wattmètre enregistreur. On trace au préalable, sur le papier diagramme de cet appareil, deux courbes parallèles, l'une reproduisant le programme à $+10 \%$, l'autre ce même programme à - $10 \%$ par exemple, à l'aide d'une encre ou d'une substance conductrice. L'aiguille de cet apareil est constituée par un stylet métallique dont la position sur le diagramme correspond exactement à la puissance réelle débitée par la machine. Lorsque ce stylet rentre en contact avec l'une des deux courbes précitées, il ferme un circuit électrique dont le courant, après amplification, agit dans le sens convenable sur le moteur de commande du régulateur de la turbine par l'intermédiaire de relais de caractéristiques appropriées; la puissance débitée par la machine se trouve ainsi réglée automatiquement sur le programme à - $+10 \%$ près.

Lorsque, conformément au programme prévu, la machine doit cesser de débiter sur le réseau, le dispositif de commande automatique agit en premier lieu sur le régulateur de la turbine dans le sens «fermeture ». A fin de course, un contact auxiliaire provoque simultanément le déclenchement du disjoncteur haute-tension, l'ouverture du circuit d'excitation, et enfin la fermeture de la vanne d'admission. La machine s'arrête.

En cas de fonctionnement de l'un des dispositifs de protection, par exemple thermomètre à contact des paliers, protection différentielle, etc..., ce dispositif provoque simul lanément le déclenchement du disjoncteur haute-tension, la coupure de l'excitation, la fermeture rapide du régulateur de la turbine et le blocage du dispositif de mise en marche automatique. En outre, s'il s'agit d'un défaut interne détecté par une protection différentielle, une pro- 
tection de masse ou une protection contre les courtscircuits entre spires, on prévoit souvent également le déclenchement simultané d'une batterie de bouteilles de $\mathrm{CO}^{2}$ (protection contre l'incendie), destinée à limiter les dégâts que peut entraîner un amorçage à l'intérieur de la machine.

La figure $\mathrm{n}^{\circ} 7$ donne, à titre d'exemple, le schéma d'avant-projet du dispositif de commande automatique d'une centrale asservie à un programme d'irrigation.

\section{INSTALLATIONS MIXTES}

Nous avons sommairement décrit, au cours de cet article, d'une part divers dispositifs de télécommande des installations électriques, d'autre part des installations entièrement automatiques.

Il est bien évident que toutes les solutions mixtes combinant la surveillance partielle, la télécommande et l'automaticité sont également réalisables.

On peut, en particulier, provoquer la mise en route automatique à distance d'une centrale par le seul fait de mettre sous tension la ligne qui relie cette centrale à une installation voisine en fermant l'interrupteur hautetension à l'arrivée de cette ligne. En ouvrant ce même interrupteur, on peut en provoquer l'arrêt complet.
Pour de petites centrales peu éloignées ou surveillées par un simple gardien, on peut effectuer manuellement la mise en route et le couplage des machines, les autres manœuvres d'exploitation courante pouvant être rendues automatiques ou effectuées par télécommande.

On peut également amener directement chaque ligne haute-tension issue d'une machine sur une installation voisine normalement surveillée et effectuer à l'arrivée les manœuvres de couplage, les autres opérations étant effectuées partie automatiquement, partie par télécommande; il devient alors possible de placer la grande majorité des mesures et contrôles à l'arrivée et économiser ainsi la plupart des télémesures.

Seule, une étude technique et financière approfondie de chaque cas particulier permettra de déterminer la meilleure solution à apporter au problème posé.

Les solutions de télécommande ou d'automaticité sont particulièrement intéressantes pour des installations de faible importance et notamment pour de petites centrales. Elles conduisent souvent à des dispositifs relativement simples et peu onéreux, et permettent d'effectuer des économies importantes du fait de la suppression presque totale du personnel spécialisé exigé par une installation normalement surveillée. 